\title{
Development of a Measurement System for Body Conduction Sounds using a Bluetooth Communication
}

\author{
Tokihiro Tamuraa, Fumiaki Ehara ${ }^{\mathrm{a}, *}$

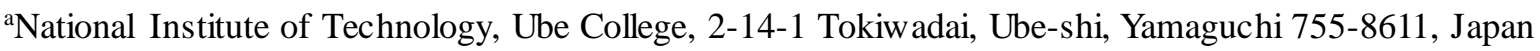 \\ *Corresponding Author: ehara@ube-k.ac.jp
}

\begin{abstract}
Solitary death of elderly people is recognized as social problem in Japan. There is a compelling need for a device that notifies the hospital at once when they have a health accident. In this study, we made a system which watched health condition by measuring a body conduction sound. The systemis comprised of measuring equipment and a receiving device. The measureing equipment consists of a microcomputer, a body conduction sound sensor and a bluetooth module. An Android smartphone is used as a receiving device. The measurement data is sent to an android terminal through bluetooth communication.
\end{abstract}

Keywords: body conduction sound, Wi-Fi, mbed.

\section{Introduction}

The average life expectancy is increased in recent years. With the increase in the number of the elderly, it is expected that a wellness is sue of them is also increased. There is a compelling need for a device that examines the health condition, notifies the hospital at once when they have a problem. It is difficult to substantiate a system which monitor them using camera because of lack of privacy. Conscious of privacy is necessary for a new monitoring system.

A sound that produced and propagated inside of the body is measured by a body conduction sound sensor that is put on the body surface. Some researchers reported a system to find unusual sounds of the heart, and an analysis method was reported that finds an abnormal cardiac sound measured by the wireless electronic stethoscope ${ }^{(1-3)}$. A system using NAM (non-audible murmur) microphones was also reported $^{(4,5)}$. NAM microphone was developed to detect a very weak speech sound. NAM microphone can be produced more cheaply than the electronic stethoscope, and has aen advantage in commercialization.

In recent years, development of microcomputers makes it possible easier and more cheaply to structure a measurement systemthan before. It is expected that a system which examines the health condition at a distant place, which diagnoses an internal disease are developed by combining the body conduction sound sensor, wireless radio communication device and a microcomputer ${ }^{(6-8)}$.

In this study we made a system that measures a body conduction sound and send the result to an Android smartphone by using a mbed microcomputer and a bluetooth communication device.

\section{Measurement System}

\subsection{System summary}

Figure 1 is system summary in this study. This system is assumed to monitoring the subjects' condition using measurement equipment. It measures heart sounds by a body conduction sound sensor, and sends the data via Bluetooth communication to an Android smartphone. The Android smartphone analyzes measurement data. If It detects anomaly, it alerts to a healthcare agency and so on.

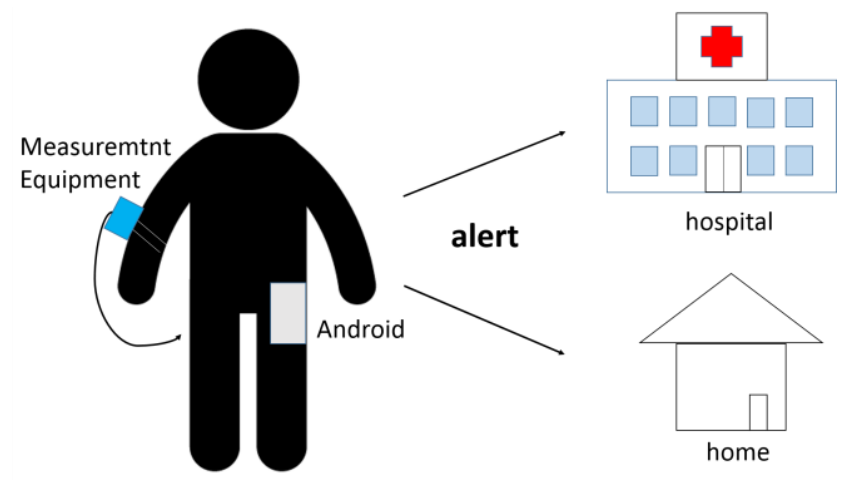

Fig. 1. Figure of system summary 


\subsection{Body conduction sound sensor}

The body conduction sound sens or is made by use of an improved small electric condenser microphone (Primo Co., EM189T). The microphone whose case is drilled a hole in and diaphragm is exposed is used. Figure 2 shows the container of the sensor that is made using acrylic as a material. The microphone is fixed in the center of the container, filled up with polyurethane resin. Then it can detect sounds inside of the body. Figure 3 is a picture of the sensor.

\section{3 mbed and Bluetooth}

Figure 4 is schematic of figure telecommunication systemin this study. This system is assumed to monitoring

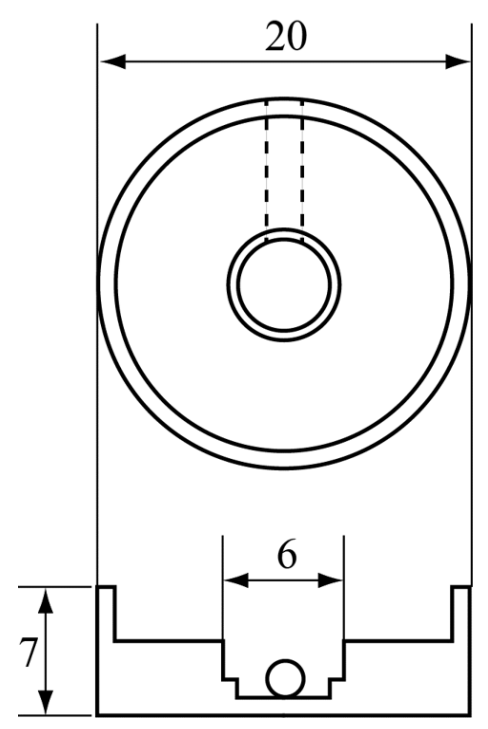

"Unit: mm"

Fig. 2. Structure of the container of the sensor.
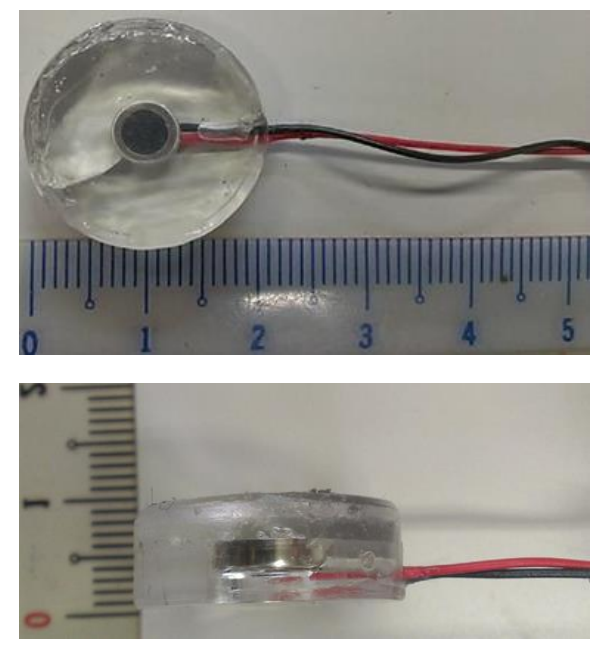

Fig. 3. The body conduction sound sensor. the subjects' condition using the sensor that mounted the microcomputer.

In this study, we use a mbed LPC1768 as a Microcomputer, it is built-in USB created by microproces sor NXP Semiconductors Company. The mbed is a microcomputer board for prototyping. Figure 5 shows the mbed LPC1768 and Table 1 shows the specifications. It is a microcontroller board based on the ARM Cortex-M3 microcontroller. It has 26 digital input-output pins and 6 analog inputs. It connects to a computer with a USB cable and we can write a program using the connection. It is an online development environment running on the browser and installation of the development environment is not required to the PC.

It was fitted with a body conduction sound sensor and Bluetooth module to mbed. We measure a heart sound by a sensor mounted on the mbed. The measured heart sound send to Android smartphone via Bluetooth. Created the Android application saves the received value in the file and depicts this data graphically.

In this study, Bluetooth is used as a means of communication. Figure 6 shows the Bluetooth module (Microchip Technology Inc, RN42-I/RM). The module is low power consumption and suitable for our system. Bluetooth can be easily used and the most populer device for wireless communication. In this study, we target the development of user-friendly system which is able to investigate of health conditions using internal sounds of the body.

$\mathrm{RN}-42-1 / \mathrm{RM}$

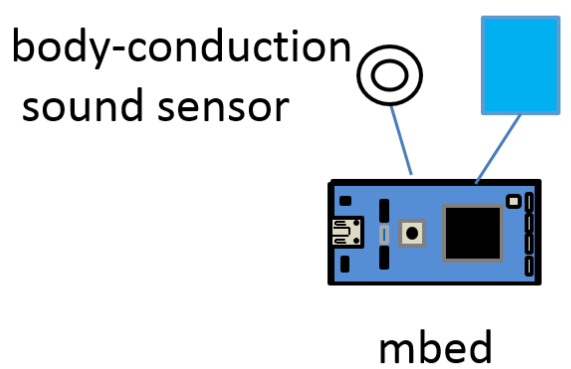

Fig. 4. Measurement equipment 


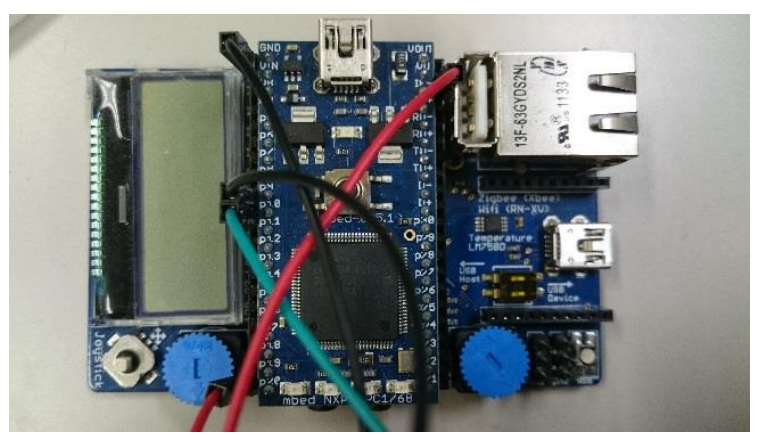

Fig.5. mbed and mbed application board

Table 1. Specifications of mbed LPC1768

\begin{tabular}{|c|c|}
\hline Core & ARM Cortex-M3 \\
\hline Frequency & $96 \mathrm{MHz}$ \\
\hline Flash Memory & $512 \mathrm{~KB}$ \\
\hline RAM & $32 \mathrm{~KB}$ \\
\hline Supply Voltage & $4.5-9.0 \mathrm{~V}$ \\
\hline Digital I/O Pins & 26 \\
\hline Analog Input Pins & 6 \\
\hline PWM Output Pins & 8 \\
\hline Analog Output Pins & 1 \\
\hline
\end{tabular}

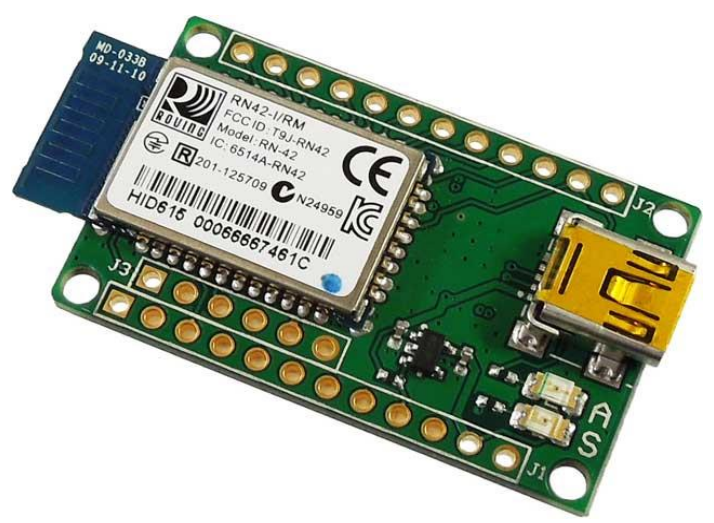

Fig. 6. RN42-I/RM

\subsection{Program of mbed}

Fig.7 is a flowchart of the mbed program. We measure a body conduction sound using the body conduction sound sensorattached to the mbed. The measured sound was send to an Android device in real time using Bluetooth communication. The baud rate is set to 115200 [bps] in the program.

\subsection{Android Application}

The values measured by the sensor connected to the mbed are transmits to an Andoroid device by Bluetooth communication. Fig 8 shows the flowchart of the Andoroid application we made. The application consists of three parts as follows.

(i) receive the measured value of body conduction sound sensor attached to the mbed via Bluetooth communication.

(ii) save the measured value in a text file.

(iii) display the measured value as a graph.

Fig 9 shows a graph displayed of the Andoroid application.

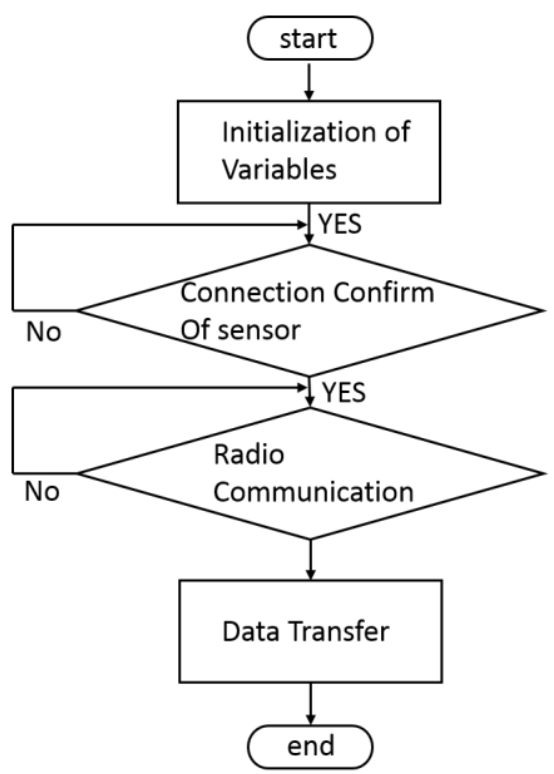

Fig.7. Flowchart to the mbed program

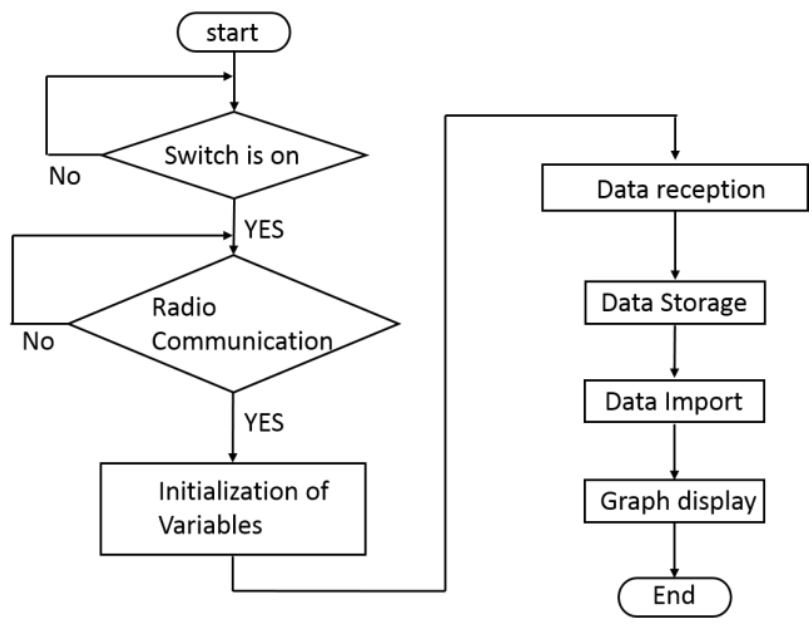

Fig.8. Flowchart of the Android Aplication 


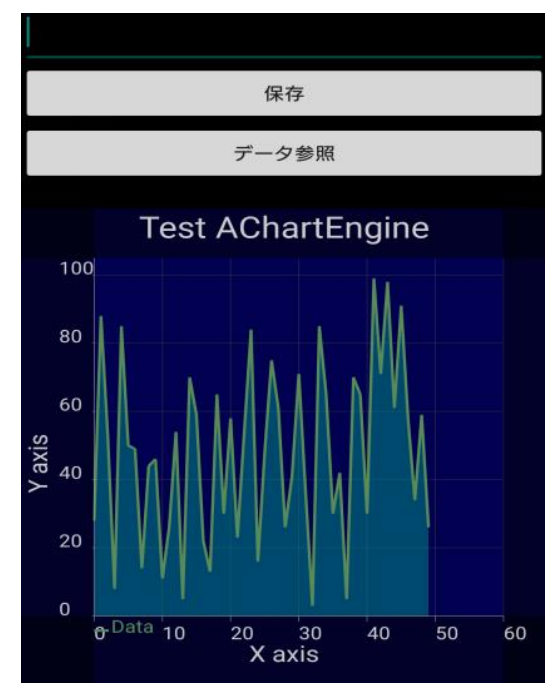

Fig.9. Android Application glaph

\section{Experiment}

We examined the system in the experiment. Android smartphone received the measured value of body conduction sound sensor via Bluetooth communication.

\section{Conclusions}

We made a system that measures a body conduction sound and send the result to an Android smartphone by using a mbed microcomputer and a bluetooth communication device. We examined the system, it can measure sounds from a sensor attached to the mbed and send to an Android smartphone via Bluetooth communication.

We want to enhance the system function to anomaly detection by body conduction sound.

\section{References}

(1) J.R. Bulgrin, B.J. Rubal, C.R. Thompson and J.M. Moody : "Comparis on of Short-Time Fourier, Wavelet and Time-Domain Analysis of Intracardiac Sounds", Biomed Sci. Instrum. Vol.29, pp.465-472, 1993

(2) C.H. Wu, C.W. Lo and J.F. Wang : "Computer-Aided Analysis and Classification of Heart Sounds Based on Neural Networks and Time Analysis", Proc. IEEE Int. Conf. Acoust. Speech Signal Process, pp.355-3458, 1995

(3) Z. Jiang and S. Choi : "Development of Wireless Electronic Stethoscope System and Abnormal Cradiac Sound Analysis Method (Sound Characteristic Waveform Analysis )", The Japan Society of Mechanical
Engineers, pp.140-147, 2005

(4) T. Hirahara and S. Shimizu : "Sensing various types sound generated inside the body using an ECM-based compact body-conducted sound sensor", Proc. Autumn Meet. Acoust. Soc. Jpn., 2009

(5) S. Shimizu, M. Otani and T. Hirahara : "Frequency characteristics of several non-audible murmur (NAM) microphones", Acoust. Sci. \& Tech., pp.139-142, 2009

(6) Fumiaki Ehara, Ryouhei Tanaka and Kanya Tanaka : "Development of a Wi-Fi-enabled Measurement System for a Body Conduction Sound", Journal of the Institute of Industrial Applications Engineers, Vol.3, No.4, pp.174-177, 2015

(7) Fumiaki Ehara, Takahiro Kubo and Kanya Tanaka : "Measurement System of Heart Rate Using a Mbed Microcontroller", INFORMATION, Vol.17 No.2, pp.545-550, 2014

(8) Fumiaki Ehara, Toshiaki Tsuruda and Kanya Tanaka : "Development of a Heart Rate Measurement Method Using Body Conduction Sound Sensor", Applied Mechanics and Materials, Vol.103, pp.616-621, 2012 